\title{
Effectiveness of gabapentin pharmacotherapy in chemotherapy-induced peripheral neuropathy
}

\author{
Magdalena Magnowska ${ }^{1}$, Natalia Iżycka ${ }^{1}$, Joanna Kapoła-Czyż ${ }^{2}$, Anna Romała ${ }^{2}$, \\ Jakub Lorek ${ }^{1}$, Marek Spaczyński ${ }^{3}$, Ewa Nowak-Markwitz ${ }^{1}$ \\ ${ }^{1}$ Department of Gynecology, Obstetrics and Gynecologic Oncology, Division of Gynecologic Oncology, \\ Poznan University of Medical Sciences, Poznan, Poland \\ ${ }^{2}$ Student's Scientific Society by the Division of Gynecologic Oncology, Poznan University of Medical Science, Poznan, Poland \\ ${ }^{3}$ The Faculty of Medicine and Health Sciences, University of Zielona Góra, Poland
}

\begin{abstract}
Objectives: Chemotherapy-induced peripheral neuropathy (CIPN) is a common chemotherapy side effect, but its prevention and treatment remains a challenge. Neurotoxicity may lead to dose limitation or even treatment discontinuation, and therefore potentially affect the efficacy of anticancer treatment and long term outcomes. The practice to administer gabapentin for neuropathy may be applicable, but is limited by insufficient studies. The aim of our study was to assess the presence of chemotherapy-induced peripheral neuropathy in ovarian cancer patients treated with first-line paclitaxel and carboplatin chemotherapy and evaluate the effectiveness of gabapentin in treatment of this condition.

Material and methods: 61 ovarian cancer patients treated with first line chemotherapy were included in the study. The first phase of the study was to assess neurological condition of each patient by: neuropathy symptoms scale, McGill's scale, neurological deficit and quality of life, during the chemotherapy. In the second phase of the study we evaluated the response to gabapentin treatment in a group of patients who developed neuropathy.

Results: $78.7 \%$ of the patients developed chemotherapy related neuropathy. During the course of chemotherapy these patients experienced significant exacerbation of neuropathy symptoms $(p<0.0001)$, neuropathic pain $(p<0.0001)$, neurologic deficit $(p<0.0012)$ and worsening of quality of life $(p<0.0002)$. Patients who were qualified to undergo the gabapentin treatment observed improvement in symptoms $(p<0.027)$, pain $(p<0.027)$ and neurologic deficit $(p<0.019)$. Quality of life did not change significantly after gabapentin treatment $(p<0.128)$.

Conclusions: Chemotherapy substantially deteriorates the neurologic condition of the patients and the quality of life. Paclitaxel and carboplatin treated patients may benefit from gabapentin therapy in chemotherapy-induced peripheral neuropathy. Key words: chemotherapy, neuropathy, McGill's scale, neurologic deficit, gabapentin
\end{abstract}

Ginekologia Polska 2018; 89, 4: 200-204

\section{INTRODUCTION}

Chemotherapy-induced peripheral neuropathy (CIPN) is a common toxicity which occurs in about $70-90 \%$ of patients treated with chemotherapy. It affects nerve function resulting in peripheral, bilateral glove and stocking paraesthesias, such as numbness, burning, tingling sensations, pain, muscle weakness and cramps. Loss of temperature sensibility may also be experienced. It is caused by sensory, rather than motor, axonal damage [1]. Most often, this type of toxicity is associated with the use of platinum agents, taxanes and vinka alkaloids. Usually the symptoms get worse as the chemotherapy is continued, but may partially or completely resolve after treatment cessation [2]. In a subset of patients the symptoms have been reported as irreversible despite the time that passed since the administration of last dose of chemotherapy [3].

Neurotoxicity induced by cytotoxic agents can adversely affect treatment and long-term outcomes leading to dose-limitation or even treatment discontinuation. Recently, more impact is put on effective support of cancer patients during and after chemotherapy due to increasing number of cancer treatment survivors and longer post treatment 
survival time. Unfortunately, neuropathy prevention and treatment still remain a big challenge. According to the latest ASCO Guidelines there are no established agents recommended for the CIPN prevention and serotonin- norepinephrine reuptake inhibitor (SNRI) duloxetine is the only drug proven to be effective and recommended in the treatment of CIPN [4]. The clinical practice to offer anticonvulsants (gabapentin), antidepressants (tricyclics) and topical capsaicin or lidocaine may be applicable, but is limited by lack of conclusive studies on CIPN treatment.

Gabapentin is an anti-epileptic drug, registered for the treatment of post-herpetic neuralgia, diabetic neuropathy, spinal cord injury pain [5] and neuropathic cancer pain [6]. Its analgesic mechanism is probably due to antagonism of the $a_{2} \delta$ calcium channels at presynaptic sites [7]. The most common side-effects are nausea, vertigo, somnolence, weight gain and asthenia. These can lead to dosage adjustment and partial reduction in neuropathic pain.

The purpose of this study was to assess the incidence of chemotherapy-induced peripheral neuropathy in ovarian cancer patients treated with first-line adjuvant paclitaxel and carboplatin chemotherapy and to evaluate the effectiveness of gabapentin in treatment of neuropathy symptoms.

\section{MATERIAL AND METHODS}

Data analysis was performed prospectively, on patients admitted to Department of Obstetrics and Gynecology due to ovarian cancer. Our study included 61 women, aged 47-63 . All of the patients were diagnosed with ovarian cancer FIGO stage III A-C and underwent initial surgical treatmentradical hysterectomy, bilateral salpingooophorectomy and omentectomy according to ovarian cancer surgical treatment protocol. All of the participants were later treated with first line paclitaxel and carboplatin chemotherapy, receiving standard doses of paclitaxel $\left(175 / \mathrm{m}^{2}\right)$ and carboplatin (according to AUC 6, but not more than $400 \mathrm{mg} / \mathrm{m}^{2}$ ). Patients underwent 6 courses of chemotherapy, every 3 weeks. The study was approved by the Institutional Review Board. The exclusion criteria was diabetes mellitus and any other potential cause for neuropathy prior to chemotherapy, such as neurologic or rheumatic diseases.

Neurological examination was performed every 3 weeks - during each hospital admission for chemotherapy. Patients were assessed according to scales that are commonly used for evaluation of neuropathy symptoms such as neuropathy symptoms scale (Tab. 1) and McGill's scale (Tab. 2). All patients underwent clinical neurological examination consisted of Achilles tendon reflex, touch sensibility (by means of standarized Semmes-Weinstein $5.07 / 10 \mathrm{~g} / \mathrm{cm}^{2} \mathrm{mo-}$ nofilament), pain sensitivity and cryaesthesia (Tab. 3). The examination was performed by qualified study team members, all of whom were medical doctors, trained to perform

\begin{tabular}{|l|c|c|}
\hline Table 1. Neuropathy symptoms scale & & \\
\hline Lower limbs & Present & Absent \\
\hline Burning sensations & 2 & 0 \\
\hline Numbness & 2 & 0 \\
\hline Paraesthesia & 2 & 0 \\
\hline Cramps & 1 & 0 \\
\hline Pain & 1 & 0 \\
\hline Muscle weakness & 1 & 0 \\
\hline Symptoms localization & & \\
\hline Foot & 2 & \\
\hline Shin & 1 & \\
\hline Other & 0 & \\
\hline Symptoms intensification & & \\
\hline Night & 2 & \\
\hline Day and night & 1 & \\
\hline During sleep & 1 & \\
\hline Day & 0 & \\
\hline Symptoms improvement & & \\
\hline During walk & 2 & \\
\hline During standing & 1 & \\
\hline During sitting or laying & & \\
\hline & & \\
\hline
\end{tabular}

\section{Table 2. McGill's neuropathic pain scale}

\begin{tabular}{|l|cccccccc|c|}
\hline Lack of pain & 0 & & & & & & & \\
\hline Low intensification pain & 1 & 2 & 3 & 4 & 5 & 6 & & \\
\hline Medium intensification pain & 7 & 8 & 9 & 10 & 11 & 12 & 13 & 14 \\
\hline Hard intensification pain & 15 & 16 & 17 & 18 & 19 & 20 & & \\
\hline
\end{tabular}

Table 3. Neurologic deficit scale

\begin{tabular}{|l|c|}
\hline Achilles tendon reflex & Points \\
\hline Within norm & 0 \\
\hline Can be developed & 1 point/side \\
\hline Cannot be developed & 2 points/sides \\
\hline Cryaesthesia & \\
\hline Within norm & 0 \\
\hline Lowered/absent & 1 point/side \\
\hline Touch sensibility & \\
\hline Within norm & 0 \\
\hline Lowered/absent & 1 point/side \\
\hline Pain sensitivity & \\
\hline Within norm & 0 \\
\hline Lowered/absent & 1 point/side \\
\hline
\end{tabular}

neurological examination. The participants were asked to fill the quality of life questionnaire during each stay, using a 10-points visual analogue scale (VAS) to determine their 
comfort of life, where 1 was very bad and 10 was very good. Each patient who developed neuropathy symptoms during the course of chemotherapy was offered gabapentin $300 \mathrm{mg}, 3$ times daily. The patients were offered maximum dose of $900 \mathrm{mg}$ per day. The minimal effective dose was approved and recommended by our consultant neurologist to minimize the possible side effects of the treatment and therefore avoid treatment discontinuation. Final neurologic examination was performed after a month of treatment with gabapentin. If the drug tolerance was good and the patients reported improvement of neuropathy symptoms, the gabapentin treatment was continued until the end of cytotoxic therapy in the same dose.

\section{Statistical analysis}

Wilcoxon test was used to compare baseline and final outcomes of the above mentioned scales and examinations. The significance level was $p<0.05$. All analyses were performed using PQstat 1.4.8.

\section{RESULTS}

In the study group of 61 patients, 48 (78.7\%) developed symptoms of neuropathy. The typical "gloves and socks" signs affected 24 (39\%) women, 20 (32.8\%) - only feet and $4(8.2 \%)$ - only hands. In a first phase of the study we assessed if our patients experienced significant worsening of the neuropathic symptoms during the chemotherapy treatment. Statistical analysis of the first phase included the time before introduction of any additional neuropathy treatment. In most patients, severe exacerbation of the neuropathic symptoms, neuropathic pain (McGill's scale) and neurologic deficit were observed after the third cycle of chemotherapy. These findings were also covered by the results of neurological examination. The deterioration of the neuropathic symptoms resulted also in the significantly worsening of quality of life (Tab. 4, Fig. 1).
In the second phase of the study we wanted to evaluate the effectiveness of gabapentin pharmacotherapy in chemotherapy related neuropathy. In the group of patients (20 women), who were qualified for gabapentin treatment, basing on significant worsening of neuropathy symptoms reported by patients or shown in the neurological examination, we performed the neurologic examination and tests before gabapentin administration (at baseline visit). The final neurologic assessment was done after a month of gabapentin treatment. Significant improvement of neuropathy symptoms, neurologic deficit and reduction of neuropathic pain was observed during the final examination. However, there was no significant change in quality of life in the study group (Tab. 5, Fig. 2). It is important to mention that 7 patients were withdrawn from the study group due to adverse effects of gabapentin. Despite that, they were all included into the study statistical analysis. The

Table 4. Assessment of neuropathy after chemotherapy, without gabapentin pharmacotherapy

\begin{tabular}{|l|c|}
\hline Type of the scale & $\mathbf{p}$ \\
\hline Neuropathy symptoms & 0.0001 \\
\hline McGill's & 0.0001 \\
\hline Neurologic deficit & 0.0012 \\
\hline Quality of life & 0.0002 \\
\hline
\end{tabular}

Table 5. Effectiveness of gabapentin pharmacotherapy, comparison between baseline visit and visit after gabapentin therapy

\begin{tabular}{|l|c|}
\hline Type of the scale & $\mathbf{p}$ \\
\hline Neuropathy symptoms & 0.027 \\
\hline McGill's & 0.027 \\
\hline Neurologic deficit & 0.019 \\
\hline Quality of life & 0.128 \\
\hline
\end{tabular}
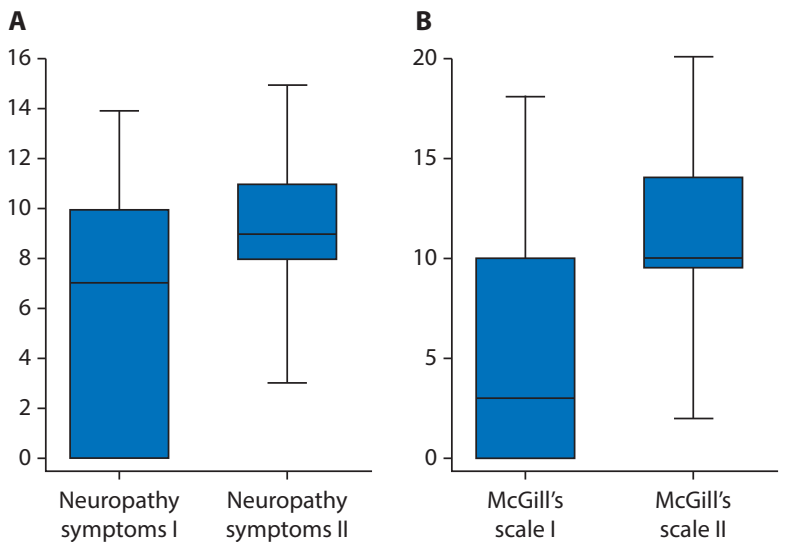

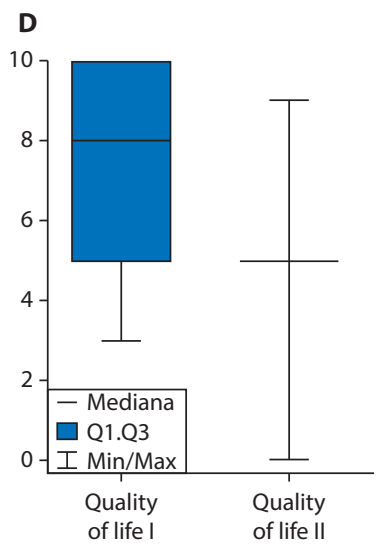

Figure 1. Comparison of visit I (when neuropathy starts) and visit II (without gabapentin treatment) A - neuropathy symptoms, B - McGill's scale, C - neurologic deficit, D - quality of life 
A

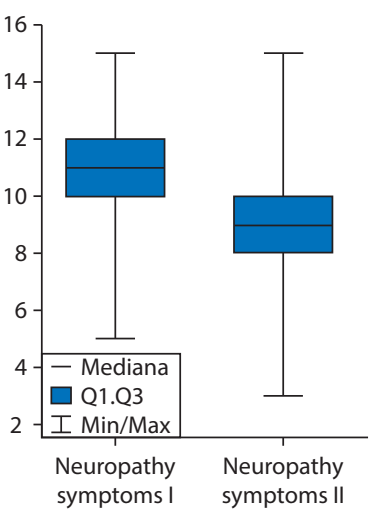

\section{B}

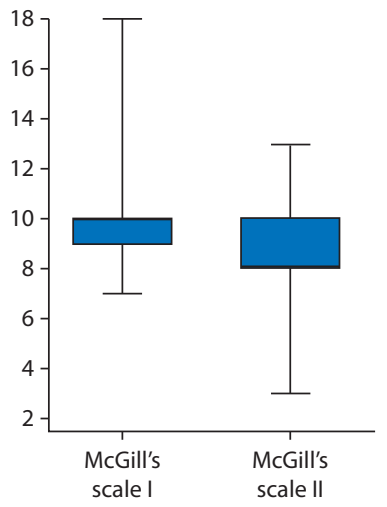

C

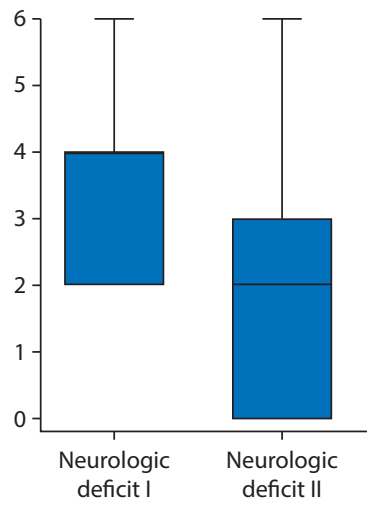

D

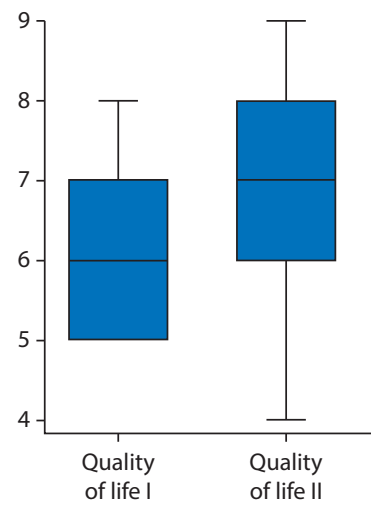

Figure 2. Comparison of visit I (without gabapentin) and visit II (after a month of gabapentin pharmacotherapy) A - neuropathy symptoms, B - McGill's scale, C - neurologic deficit, D - quality of life

main complaint was on vertigo and side effects from the gastrointestinal tract.

\section{DISCUSSION}

Chemotherapy-induced peripheral neuropathy is a common adverse effect which affects long-term quality of life and may potentially influence long-term outcomes of anti-cancer treatment. The overall incidence depends on the type and dose of cytostatic drug and the duration of the treatment. Any other patient's dependent risk factors are not known. The incidence of CIPN according to our study $-78.8 \%$ is consistent with another surveys $[8,9]$. The importance of the problem should not be underestimated, because the neuropathy symptoms may not only persist for up to 12 years and seriously affect quality of life [8], but also influence the long term treatment outcomes causing chemotherapy dose reductions. Patients expect effective cancer treatment, as well as the side effect reducing supportive therapy. Gabapentin is a drug which has been studied in a treatment of diabetic neuropathy and post herpetic neuralgia. The 2013 Cochrane Database overview of 29 studies on over 3000 patients, provided some evidence of gabapentin efficacy. This drug reduced the neuropathic pain by half, in $10-25 \%$ of the patients, without any improvement in the remaining group of patients. About $25 \%$ of patients had to stop taking the drug, because of bad tolerance [9].

We also observed side effects of gabapentin, which contributed to therapy cessation in 35\% of patients. Our research of Cochrane database did not reveal any reviews on the use of gabapentin in chemotherapy induced neuropathy. The only reliable recommendations for prevention and management of CIPN were published by ASCO. These Guidelines comprise 48 randomized controlled trials. According to that analysis none of the following drugs and supplements should be recommended for CIPN prevention: acetyl-L-carnitine, amitriptyline, $\mathrm{Ca}, \mathrm{Mg}$, glutathione, nimodipine, retinoic acid and vitamin $\mathrm{E}$, due to the lack of conclusive studies supporting their use in CIPN prophylaxis.

Selective serotonin and norepinephrine reuptake inhibitor (SNRI) duloxetine is the only drug proven to be effective in CIPN treatment. Although the data available for gabapentin is limited, the panel of specialists found it reasonable to try this drug, because of unmet need for CIPN treatment and the relative safety of the therapy [10]. There was only one negative randomized trial on 115 patients, which compared gabapentin in dose $2700 \mathrm{mg}$ for 6 weeks with placebo. This study showed no benefit from using gabapentin in CIPN treatment [11]. The major limitation of this study appeared to be the heterogeneous group of patients, treated with different chemotherapeutics (paclitaxel, docetaxel, carboplatin, cisplatin, oxaliplatin, vincristine and vinblastine). As the exact pathophysiology of CIPN is not explained, it is suggested that each cytostatic agent has a different mechanism of nerve damage [12]. It is hypothesized that the treatment should also be specific for each chemotherapeutic agent [13]. Our study group was much more balanced as it involved only women treated for ovarian cancer with standard doses of paclitaxel $\left(175 \mathrm{mg} / \mathrm{m}^{2}\right)$ and carboplatin (acc. AUC 6). On the other hand, the important limitation of our analysis was a relatively small study group. However, we obtained significant outcomes concerning the improvement in neurological examination, neuropathic symptoms and neuropathic pain, the quality of life remained on the same level. The lack of quality of life improvement can be explained by relatively large amount of side-effects of gabapentin. This is even more surprising according to the fact that in our study the maximum administered dose of gabapentin was $900 \mathrm{mg}$ per day, which is much lower comparing to other studies involving gabapentin. 


\section{CONCLUSIONS}

Chemotherapy substantially deteriorates the neurologic condition of the patients and the quality of life. Paclitaxel and carboplatin treated patients may benefit from gabapentin therapy in chemotherapy-induced peripheral neuropathy.

\section{Conflict of interests}

None declared.

\section{REFERENCES}

1. Argyriou AA, Bruna J, Marmiroli P, et al. Chemotherapy-induced peripheral neurotoxicity (CIPN): an update. Crit Rev Oncol Hematol. 2012; 82(1): 51-77, doi: 10.1016/j.critrevonc.2011.04.012, indexed in Pubmed: 21908200.

2. Iżycki D, Niezgoda AA, Kaźmierczak M, et al. Chemotherapy-induced peripheral neuropathy-diagnosis, evolution and treatment. Ginekol Pol. 2016; 87(7): 516-521, doi: 10.5603/GP.2016.0036, indexed in Pubmed: 27504945.

3. Tanabe $Y$, Hashimoto $K$, Shimizu C, et al. Paclitaxel-induced peripheral neuropathy in patients receiving adjuvant chemotherapy for breast cancer. Int J Clin Oncol. 2013; 18(1): 132-138, doi: 10.1007/s10147-011-0352-x, indexed in Pubmed: 22105895.

4. Hershman DL, Lacchetti C, Loprinzi CL, et al. Prevention and management of chemotherapy-induced peripheral neuropathy in survivors of adult cancers: American Society of Clinical Oncology clinical practice guideline. J Clin Oncol. 2014; 32(18): 1941-1967, doi: 10.1200/JCO.2013.54.0914, indexed in Pubmed: 24733808.

5. Finnerup NB, Sindrup SH, Jensen TS. The evidence for pharmacological treatment of neuropathic pain. Pain . 2010; 150(3): 573-581, doi: 10.1016/j.pain.2010.06.019, indexed in Pubmed: 20705215.
6. Mishra S, Bhatnagar S, Goyal GN, et al. A comparative efficacy of amitriptyline, gabapentin, and pregabalin in neuropathic cancer pain: a prospective randomized double-blind placebo-controlled study. Am J Hosp Palliat Care. 2012; 29(3): 177-182, doi: 10.1177/1049909111412539, indexed in Pubmed: 21745832.

7. Sills GJ. The mechanisms of action of gabapentin and pregabalin. Curr Opin Pharmacol. 2006; 6(1): 108-113, doi: 10.1016/j.coph.2005.11.003, indexed in Pubmed: 16376147.

8. Park SB, Kwok JB, Loy CT, et al. Paclitaxel-induced neuropathy: potential association of MAPT and GSK3B genotypes. BMC Cancer. 2014; 14: 993 , doi: 10.1186/1471-2407-14-993, indexed in Pubmed: 25535399.

9. Ezendam NPM, Pijlman B, Bhugwandass C, et al. Chemotherapy-induced peripheral neuropathy and its impact on health-related quality of life among ovarian cancer survivors: results from the population-based PROFILES registry. Gynecol Oncol. 2014; 135(3): 510-517, doi: 10.1016/j. ygyno.2014.09.016, indexed in Pubmed: 25281491.

10. Hershman DL, Lacchetti C, Loprinzi CL, et al. Prevention and management of chemotherapy-induced peripheral neuropathy in survivors of adult cancers: American Society of Clinical Oncology clinical practice guideline. J Clin Oncol. 2014; 32(18): 1941-1967, doi: 10.1200/JCO.2013.54.0914, indexed in Pubmed: 24733808.

11. Rao RD, Michalak JC, Sloan JA, et al. Efficacy of gabapentin in the management of chemotherapy-induced peripheral neuropathy: a phase 3 randomized, double-blind, placebo-controlled, crossover trial (NOOC3). Cancer. 2007; 110(9): 2110-2118, doi: 10.1002/cncr.23008, indexed in Pubmed: 17853395.

12. Cata JP, Weng HR, Lee BN, et al. Clinical and experimental findings in humans and animals with chemotherapy-induced peripheral neuropathy Minerva Anestesiol. 2006; 72(3): 151-169, indexed in Pubmed: 16493391.

13. Park HJ. Chemotherapy induced peripheral neuropathic pain. Korean J Anesthesiol. 2014; 67(1): 4-7, doi: 10.4097/kjae.2014.67.1.4, indexed in Pubmed: 25097731 